\title{
Tacrolimus as an adjunct to autologous minced muscle grafts for the repair of a volumetric muscle loss injury
}

\author{
Benjamin T. Corona, Jessica C. Rivera, Joseph C. Wenke and Sarah M. Greising ${ }^{*}$
}

\begin{abstract}
Background: Volumetric muscle loss (VML) following extremity orthopaedic trauma or surgery results in chronic functional deficits and disability. A current translational approach to address the devastating functional limitations due to VML injury is the use of an autologous minced muscle graft $\left(\sim 1 \mathrm{~mm}^{3}\right.$ pieces of muscle tissue) replacement into the injured defect area, although limitations related to donor site morbidity are still unaddressed. This study was designed to explore adjunct pharmacological immunomodulation to enhance graft efficacy and promote muscle function following VML injury, and thereby reduce the amount of donor tissue required.

Findings: Using a validated VML porcine injury model in which $20 \%$ of the muscle volume was surgically removed, this study examined muscle function over 3 months post-VML injury. In vivo isometric torque of the peroneus teritus (PT) muscle was not different before surgery among sham, non-repaired, non-repaired with tacrolimus, graft-repaired, and graft-repaired with tacrolimus VML groups. Bi-weekly torque analysis of the VML injured musculature presented a significant strength deficit of $\sim 26 \%$ compared to pre-injury in the non-repaired, non-repaired with tacrolimus, and graft-repaired groups. Comparatively, the strength deficit in the graft-repair with systemic tacrolimus was marginally improved ( 19\%; $p=0.056)$. Both of the minced graft repaired groups presented a greater proportion of muscle tissue in full-thickness histology specimen.
\end{abstract}

Conclusions: We demonstrate that adjunctive use of tacrolimus with an $\sim 50 \%$ minced muscle graft replacement resulted in modest improvements in muscle function 3 months after injury and repair, but the magnitude of improvement is not expected to elicit clinically meaningful functional improvements.

Keywords: Neuromuscular strength, Orthopaedic trauma, Porcine, Skeletal muscle injury

\section{Introduction}

Extremity orthopaedic trauma that results in volumetric muscle loss (VML) presents chronic and persistent functional muscle deficits, restricted joint range of motion, and fibrosis, which ultimately manifest as chronic disability (Mase et al. 2010; Corona et al. 2015; Garg et al. 2015; Rivera and Corona 2016). Moreover, VML injury may impair healing of concomitantly fractured bone (Willett et al. 2013; Hurtgen et al. 2016; Pollot et al. 2016; Hurtgen et al. 2017a, 2017b). In such cases where composite bone and skeletal muscle tissue injuries exist, clinical care emphasizes fracture healing with little

\footnotetext{
* Correspondence: sarah.m.greising2.ctr@mail.mil; smgreising@msn.com Extremity Trauma and Regenerative Medicine Task Area, United States Army Institute of Surgical Research, 3698 Chambers Pass, BHT1, Fort Sam Houston, TX 78234, USA
}

attention to regeneration of the traumatized skeletal musculature. Recent basic and translational research efforts are aimed at promoting de novo regeneration of the muscle tissue to ameliorate musculoskeletal regeneration and to promote long-term improvements in muscle and limb function (Corona and Greising 2016).

In both small and large animal models treatment of VML injuries with autologous minced muscle grafts $\left(\sim 1 \mathrm{~mm}^{3}\right.$ pieces of muscle tissue) have the ability to improve muscle function (Corona et al. 2013; Garg et al. 2014; Li et al. 2014; Aurora et al. 2015; Ward et al. 2015; Kasukonis et al. 2016; Ward et al. 2016). Autologous minced muscle grafts are capable of spontaneous de novo muscle fiber regeneration due to their composition of essential components to skeletal muscle regeneration, such as basal lamina and myogenic stem cells 
(Carlson 1972; Caldwell et al. 1990; Lepper et al. 2011). However, salient limitations of this approach for the repair of clinically relevant VML defects are the availability and morbidity associated with harvesting autologous muscle tissue, the rate of vascularization and innervation of regenerating muscle fibers, and the protracted pro-inflammatory wound environment that persists within VML injured skeletal muscle, which inhibits myogenic while activating fibrotic transcriptional programs (Hurtgen et al. 2016; Sadtler et al. 2016).

This study was designed to directly interrogate the impact of tacrolimus, an US Food and Drug Administration (FDA) approved immunomodulatory drug commonly used in transplant surgery, on functional regeneration mediated by autologous minced muscle graft repair of VML injury in a porcine model. Tacrolimus is a calcineurin phosphatase inhibitor that inhibits downstream Il-2 mediated activation of T lymphocytes and attenuates macrophage and dendritic cell activity (Thomson et al. 1995). Additionally, tacrolimus has neurotrophic properties that may accelerate axonal regeneration and muscle fiber reinnervation (Gold 1997; Sulaiman et al. 2002). As such, it was hypothesized that functional regeneration mediated by autologous minced muscle grafts would be augmented by adjunctive systemic delivery of tacrolimus.

\section{Methods}

\section{Study design}

Female Yorkshire Cross swine $(n=7)$ were purchased form Midwest Research Swine (Gibbon MN, USA). At the terminal experiment animals were $6.1 \pm 0.2$ months of age and considered to be young-adult (Table 1). All swine underwent initial functional testing and repeated testing every 2 weeks following surgery. At the time of surgery bilateral VML injuries (or sham procedures) were performed and then each limb was randomized to no repair or repair with a $\sim 50 \%$ volume replacement using an autologous minced muscle graft harvested from the contralateral (i.e., non-repaired) limb (Ward et al. 2016). Immediately following surgery, each swine was further randomized to treatment groups, specifically 1 month of immunomodulation using tacrolimus or no treatment. The final functional data was examined at 12 weeks post-surgery and sampling of the muscles of the hindlimb was conducted. The primary assessment of these experimental groups was in vivo isometric torque, which was analyzed using a repeated measures approach. All protocols and animal care guidelines were approved by the Institutional Animal Care and Use Committee at the United States Army Institute of Surgical Research (Protocol \#A14-018), in compliance with the Animal Welfare Act, the Implementing Animal Welfare Regulations and in accordance with the principles of the Guide for the Care and Use of Laboratory Animals.

\section{Surgical procedures}

As previously described (Pollot and Corona, 2016; Greising et al. 2017), swine were anesthetized with Telazol (4$6 \mathrm{mg} / \mathrm{kg}$, i.m.), intubated and maintained under isoflurane anesthesia. Under appropriate aseptic conditions, a $10 \mathrm{~cm}$ incision was made centered over the anterior compartment to expose the peroneus tertius (PT) muscle. The anterior aspect of the muscle was measured and a $3 \times 3 \mathrm{~cm}^{2}$ area was marked in the middle third of the PT muscle. Then this area of muscle $(5.59 \pm 0.04 \mathrm{~g})$ was sharply

Table 1 Characteristics across study period

\begin{tabular}{|c|c|c|c|c|c|}
\hline & Sham & VML & $\mathrm{VML}+\mathrm{MMG}$ & VML + Tacrolimus & $\mathrm{VML}+\mathrm{MMG}+$ Tacrolimus \\
\hline $\mathrm{n}=$ & 1 & 2 & & 4 & \\
\hline \multicolumn{6}{|c|}{ Body mass (kg) } \\
\hline Week 0 & $39.4 \pm 0.0$ & $41.9 \pm 2.3$ & & $41.7 \pm 0.8$ & \\
\hline Week 4 & $48.6 \pm 0.0$ & $49.9 \pm 3.1$ & & $46.5 \pm 0.7$ & \\
\hline Week 8 & $54.2 \pm 0.0$ & $56.6 \pm 2.8$ & & $51.8 \pm 2.8$ & \\
\hline Week 12 & $61.2 \pm 0.0$ & $65.3 \pm 3.9$ & & $55.7 \pm 3.3$ & \\
\hline \multicolumn{6}{|c|}{ Tacrolimus concentration $(\mathrm{ng} / \mathrm{ml})$} \\
\hline Day 3 & nd & nd & & $1.12 \pm 0.29$ & \\
\hline Day 9 & nd & nd & & $1.35 \pm 0.14$ & \\
\hline Week 2 & nd & nd & & $1.73 \pm 0.25$ & \\
\hline Week 3 & nd & nd & & $2.26 \pm 0.65$ & \\
\hline Week 4 & nd & nd & & $3.25 \pm 1.58$ & \\
\hline Week 6 & nd & nd & & nd & \\
\hline
\end{tabular}

Circulating levels of tacrolimus were analyzed; levels were non-detectible (nd) in the untreated and sham animals and 2 weeks following termination of treatment Mean \pm standard error

VML volumetric muscle loss, MMG minced muscle graft 
dissected from the PT muscle. Sham operated limbs were surgically exposed and the PT muscle was isolated, but no tissue was removed. A subset of VML defects were treated with autologous minced muscle grafts as previously described (Pollot et al. 2016; Ward et al. 2016). Briefly using the muscle from the contralateral VML injury an autologous minced muscle graft was created. Under sterile conditions the muscle was minced to $\sim 1 \mathrm{~mm}^{3}$ pieces and $\sim 50 \%$ volume was transplanted into the defect of the designated repair limb. To accommodate the autograft into the defect, the epimysium layer superficial to the muscle was closed over autograft with $\sim 8$ interrupted proline sutures. In all cases the fascial and skin incisions were closed in layers with absorbable suture and compressive bandages were wrapped around each limb. Post-surgical analgesic administration of Buprenorphine SR (0.01 mg/kg, s.c.), Rimadyl (4.4 mg/kg, s.c.), and Excede $(5.0 \mathrm{mg} / \mathrm{kg}$, s.c.) was conducted through 1 week post-surgically.

\section{Immunomodulatory treatment}

For the first month following surgery animals randomized to the tacrolimus immunomodulation group were administered $0.075 \mathrm{mg} / \mathrm{kg}$ daily (Prograft, Astellas Pharma Inc.; Northbrook IL, USA). Daily administration ranged from 3 to $4 \mathrm{mg}$ during the treatment period to account for body weight; all treated and non-treated swine gained approximately the same amount of weight per week. The selected dosage is recommended for cardiac transplant surgery and has been shown to result in effective serum levels of tacrolimus of $10-20 \mathrm{ng} / \mathrm{ml}$ (Podesser et al. 2005); notably whole blood concentrations can be variable as treatment begins. Evaluation of the whole blood concentrations of tacrolimus was completed by HPLC-tandem mass spec at day 3, 9, 14, 28, and 42 post-surgery, using blood collected in EDTA anti-coagulant coated tubes. No adverse events related to tacrolimus treatment were observed.

\section{In vivo functional testing}

As previously described and validated (Ward et al. 2016; Greising et al. 2017), the strength of the dorsiflexor muscles of the anterior compartment was by subdermal stimulation of the peroneal nerve using needle electrodes over a range of joint angles $\left(0-50^{\circ}\right)$. Maximal isometric tetanic torque was elicited using $100 \mathrm{~Hz}, 0.1 \mathrm{~ms}$ pulse, over $800 \mathrm{~ms}$ (Grass S88 stimulator and 890A Aurora Scientific; Ontario Canada). Torque was normalized to body weight assessed immediately prior to each procedure.

\section{Histological analyses}

The entire PT muscle was excised and muscle samples were subsequently excised using an $8 \mathrm{~mm}$ biopsy punch through the full thickness of the muscle, following the terminal in vivo functional assessment. Muscle sections were frozen in melting isopentane and stored at $-80{ }^{\circ} \mathrm{C}$ until processing and analysis. Sections were treated processing using standard histologic procedures, with each section being cross-sectioned at $8 \mu \mathrm{m}$ and stained using Masson's trichrome. Bright field images were acquired with a Zeiss Axio Scan.Z1 (Carl Zeiss Microscopy, Thornwood NY, USA) and composite images were saved separately as a 24-bit, 96 dpi color images. For display purposes only images were produced in Adobe Photoshop (Adobe Systems Inc.; San Jose CA, USA) by down-converting, without introducing any changes in brightness or contrast. During all imaging and investigation, investigators were blinded to experimental group.

\section{Statistical analyses}

All data was analyzed using JMP (version 10.0 SAS Institute, Inc., Cary NC, USA). The primary assessment (isometric torque) was analyzed using repeated measures ANOVA. Blood levels of tacrolimus were analyzed using one way-ANOVA. When appropriate Tukey HSD posthoc analysis was performed. Data are reported as mean \pm standard error, and significance was determined at the $\alpha<0.05$ level.

\section{Findings}

All animals recovered promptly following surgery and within $24 \mathrm{~h}$ displayed normal mobility and cage activity. Additionally no adverse events occurred for the duration of the study. Circulating whole blood levels of tacrolimus in treated animals was $\sim 3.3 \mathrm{ng} / \mathrm{ml}$ by 4 weeks post-surgery and was no longer detectible 2 weeks later (Table 1 ).

\section{PT muscle histology}

Histological examination of the PT muscle indicated significant fibrotic deposition overlaying the remaining muscle across all treatment groups (Fig. 1). The fibrous tissue was comprised of a mixture of immature and mature connective tissue matrix, with the more mature fibrosis indicated by dense collagen fibers with trabecular meshwork. In all cases the muscle remaining after VML injury appeared healthy, without noteworthy signs of degeneration. Sections from PT muscles that were repaired with a muscle graft presented noticeably lesser fibrotic tissue and greater muscle tissue than non-repaired VML injured muscles, regardless of tacrolimus delivery.

\section{In vivo anterior crural muscle strength}

Repeated evaluation of maximally stimulated peak isometric torque of the anterior compartment was conducted immediately before and every 2 weeks following VML injury by maximally stimulating the peroneal nerve (Fig. 2). Prior to surgery, isometric torque was not 


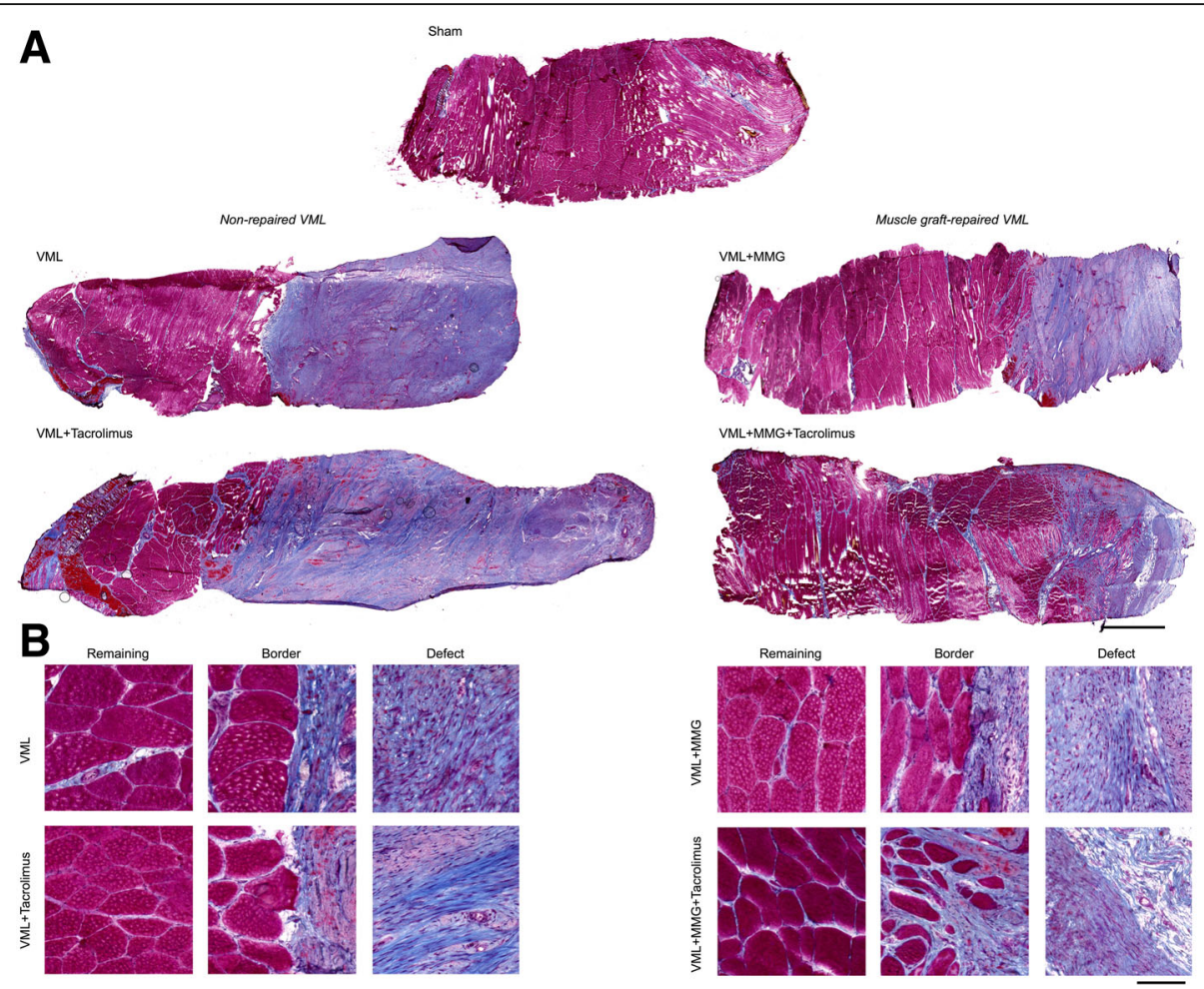

Fig. 1 Representative histologic micrographs of Masson's Trichrome stained (connective tissue is blue; nuclei are purple; skeletal muscle fibers are red) porcine peroneous tertius (PT) muscle following VML injury and repair. While all PT muscles indicate gross fibrosis following VML injury, only the muscle graft-repaired displayed areas of likely regenerated fibers. There were no apparent differences due to treatment with tacrolimus. (a) Each sample represents a full thickness sample through the muscle. Scale bar is $2 \mathrm{~mm}$; all images are at the same magnification. (b) Representative inserts from the remaining muscle, border, and defect area of the samples are displayed. Scale bar is $100 \mu \mathrm{m}$; all images are at the same magnification

different among groups $(p=0.542)$. As expected, shamoperated limbs presented consistent torque production over the 12 week study $(p=0.121)$. Non-repaired (i.e., no minced grafts) VML-injured muscles presented an average $\sim 28 \%$ isometric torque deficit from 2 to 12 weeks post-injury, which was not ameliorated by tacrolimus administration ( $24 \%$ torque deficit). Similarly, minced graft repair without tacrolimus presented a $\sim 29 \%$ functional deficit. However, the inclusion of tacrolimus delivery with minced graft repair reduce the functional deficit by approximately one-third to $\sim 19 \%$. The functional deficit for the minced graft with tacrolimus trended to be
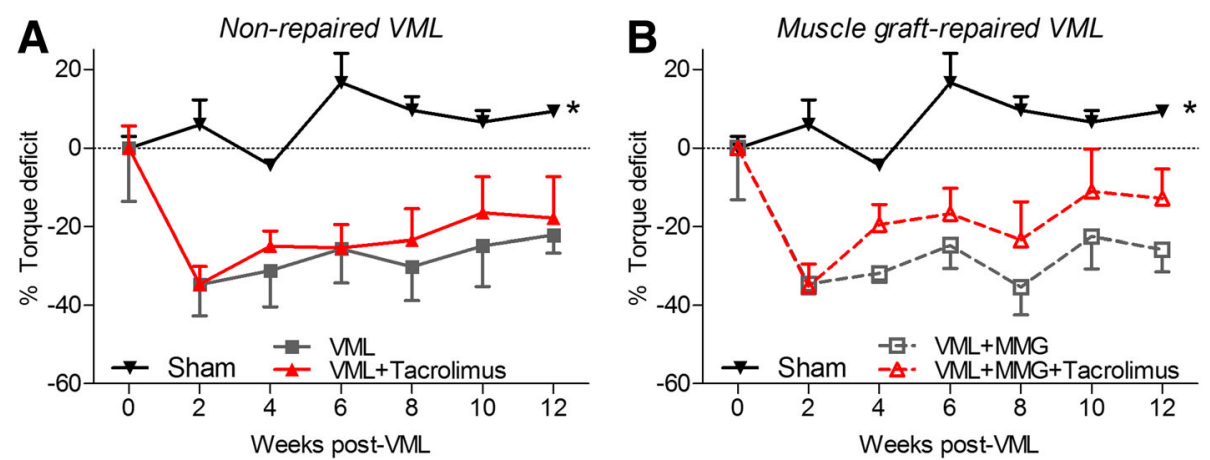

Fig. 2 Peroneus teritus (PT) muscle function was determined by peroneal nerve stimulation in vivo. The percent torque deficit to the pre-injury torque was determined for the (a) non-repaired and (b) muscle graft (MMG) repaired groups following VML injury and the effect of adjunctive tacrolimus was investigated across these groups. The strength of the sham operated group was consistent over time (duplicated in each panel) and all VML injured groups were less than sham operated. All data analyzed by two-way ANOVA (group $p<0.001$; time $p=0.104$; interaction $p=0.999)$. * Significant main effect of group, sham $>$ all other surgical groups. Data are mean \pm standard error 
statistically different from non-repaired VML injured muscle without tacrolimus $(p=0.088)$ and minced muscle graft repaired VML injured muscle $(p=0.056)$.

\section{Discussion}

We demonstrate that adjunct tacrolimus treatment to autologous minced muscle grafts tended to ameliorate neuromuscular strength deficits following VML injury. However, the data do not support a clinically meaningful therapeutic benefit using the current dosing regimen. Autologous minced muscle grafts provide necessary endogens elements of skeletal muscle necessary for repair, importantly including satellite cells. This approach does not require FDA approval as the grafts are minimally manipulated tissue. However, a current clinical limitation of autologous minced muscle grafts is the donor site burden; as such we investigated a more clinically feasible replacement volume.

Herein we delivered systemic tacrolimus for 1 month following VML injury, during a period in which myogenesis is expected to be increased. The target dosage of systemic tacrolimus was selected as it has been previously used for successful cardiac transplant (Podesser et al. 2005). While the circulating concentrations of tacrolimus were slightly below the expected range it is still expected that systemic delivery of tacrolimus presumably suppressed the immune response within the VML injured muscle. Given the exacerbated immune response that follows VML injury (Hurtgen et al. 2016) and the known detrimental impact that prolonged inflammation has on myogenesis (Tidball 2005), the original hypothesis that immunomodulation can ameliorate functional regeneration remains plausible. Though, clearly further testing is necessary to identify either an effective VML-injury specific dosing regimen for tacrolimus or a different drug that more specifically targets influential targets of the immune response to VML injury. Towards that end, heightened and prolonged macrophage presence and proinflammatory activity has been observed in rodent trauma models that involve VML injury (Hurtgen et al. 2016; Hurtgen et al. 2017a, 2017b), and thus investigation of targeted macrophage modulation may prove effective.

It was unexpected that the autologous minced muscle graft repair alone did not improve muscle strength. Previously, a $\sim 100 \%$ autologous minced muscle graft repair of VML injury that resulted in $\sim 32 \%$ strength increase compared to non-repaired VML injuries in a large animal model (Ward et al. 2016). In the current study, only $50 \%$ of the mass of the VML defect was replaced with an autologous minced muscle grafts and therefore it may be that in this large animal model a lower threshold for repair was crossed. Prior observations in which a 50 and $100 \%$ minced graft repair achieved similar levels of muscle fiber regeneration and strength recovery in a rat tibialis anterior muscle VML model supported the use of this level of repair in the current study. Somewhat encouraging, however, was the obvious greater proportion of skeletal muscle tissue in the minced graft repair versus the non-repaired groups. It is expected that this proportion of muscle fibers is due to de novo regeneration of fibers, which has been previously validated in rodent models (Ward et al. 2015; Corona et al. 2017). It is possible that the minced grafts did indeed promote skeletal muscle regeneration but that the fibers were not yet innervated. In that scenario, the tendency for improved strength in the minced graft group with adjunct tacrolimus administration may reflect the compounds (i.e., FK506) beneficial impact on peripheral nerve regeneration (Gold 1997; Sulaiman et al. 2002).

\section{Conclusion}

The current study demonstrates that delivery of tacrolimus as an adjunct to autologous minced graft repair of VML injury has the potential to reduce prolonged muscle functional deficits. Notably, delivery of $\sim 50 \%$ minced grafts to the VML without adjunctive immunomodulation did not promote recovery of strength, but did appear to promote muscle tissue regeneration. These findings highlight the need for further studies to delineate an effective immunomodulatory approach to augment minced muscle grafts in the repair of VML injury; such studies may be best suited to a standardized rodent VML model.

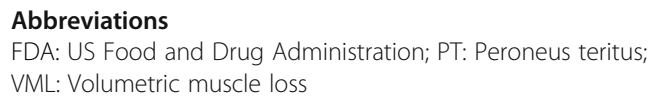

\section{Acknowledgements}

The authors gratefully acknowledge the USAISR Veterinary Support and Comparative Pathology Branches, and Mrs. Monica Jalomo for technical assistance in the completion of these studies. Studies were funded by the US Army Medical Research and Material Command, Clinical and Rehabilitative Medical Research Program (Award \#C_003_2015_USAISR to BTC).

\section{Availability of data and materials}

The datasets used and/or analyzed during the current study are primarily presented in the current manuscript and are available from the corresponding author on reasonable request.

\section{Disclosures}

The opinions or assertions contained here are the private views of the authors and are not to be construed as official or as reflecting the views of the Department of the Army, the Department of Defense, or the United States Government.

\section{Authors' contributions}

BTC, JCW, and SMG designed the study. BTC, JCR, and SMG performed experiments and collected data. BTC and SMG analyzed the data, and wrote the manuscript. All authors have read and approved the final version of this manuscript.

\section{Ethics approval}

All animal procedures were approved by the Institutional Animal Care and Use Committee of the United States Army Institute of Surgical Research (A-14-018) and were conducted in compliance with the Animal Welfare Act and in accordance with the principles of the Guide for the Care and Use of Laboratory Animals. 


\section{Competing interests}

The authors declare that they have no competing interests.

\section{Publisher's Note}

Springer Nature remains neutral with regard to jurisdictional claims in published maps and institutional affiliations.

Received: 13 July 2017 Accepted: 20 October 2017

Published online: 10 November 2017

\section{References}

Aurora A, Roe JL, Corona BT, Walters TJ (2015) An acellular biologic scaffold does not regenerate appreciable de novo muscle tissue in rat models of volumetric muscle loss injury. Biomaterials 67:393-407

Caldwell CJ, Mattey DL, Weller RO (1990) Role of the basement membrane in the regeneration of skeletal muscle. Neuropathol Appl Neurobiol 16(3):225-238

Carlson BM (1972) The regeneration of minced muscles. S. Karger, Basel, New York

Corona BT, Greising SM (2016) Challenges to acellular biological scaffold mediated skeletal muscle tissue regeneration. Biomaterials 104:238-246

Corona BT, Garg K, Ward CL, McDaniel JS, Walters TJ, Rathbone CR (2013) Autologous minced muscle grafts: a tissue engineering therapy for the volumetric loss of skeletal muscle. Am J Physiol Cell Physiol 305(7):C761-C775

Corona BT, Rivera JC, Owens JG, Wenke JC, Rathbone CR (2015) Volumetric muscle loss leads to permanent disability following extremity trauma. J Rehabil Res Dev 52(7):785-792

Corona BT, Henderson BE, Ward CL, Greising SM (2017) Contribution of minced muscle graft progenitor cells to muscle fiber formation after volumetric muscle loss injury in wild-type and immune deficient mice. Physiol Rep 5(7): e13249. doi:10.14814/phy2.13249

Garg K, Ward CL, Rathbone CR, Corona BT (2014) Transplantation of devitalized muscle scaffolds is insufficient for appreciable de novo muscle fiber regeneration after volumetric muscle loss injury. Cell Tissue Res 358(3):857-873

Garg K, Ward CL, Hurtgen BJ, Wilken JM, Stinner DJ, Wenke JC, Owens JG, Corona BT (2015) Volumetric muscle loss: persistent functional deficits beyond frank loss of tissue. J Orthop Res 33(1):40-46

Gold BG (1997) FK506 and the role of immunophilins in nerve regeneration. Mol Neurobiol 15(3):285-306

Greising SM, Rivera JC, Goldman SM, Watts A, Aguilar CA, Corona BT (2017) Unwavering Pathobiology of volumetric muscle loss injury. Sci Rep 7(1):13179

Hurtgen BJ, Ward CL, Garg K, Pollot BE, Goldman SM, McKinley TO, Wenke JC, Corona BT (2016) Severe muscle trauma triggers heightened and prolonged local musculoskeletal inflammation and impairs adjacent tibia fracture healing. J Musculoskelet Neuronal Interact 16(2):122-134

Hurtgen BJ, Henderson BEP, Ward CL, Goldman SM, Garg K, McKinley TO, Greising SM, Wenke JC, Corona BT (2017a) Impairment of early fracture healing by skeletal muscle trauma is restored by FK506. BMC Musculoskelet Disord 18(1):253

Hurtgen BJ, Ward CL, Leopold Wager CM, Garg K, Goldman SM, Henderson BEP, McKinley TO, Greising SM, Wenke JC, Corona BT (2017b) Autologous minced muscle grafts improve endogenous fracture healing and muscle strength after musculoskeletal trauma. Physiol Rep 5(14):e13362. doi:10.14814/phy2. 13362

Kasukonis B, Kim J, Brown L, Jones J, Ahmadi S, Washington T, Wolchok J (2016) Codelivery of infusion Decellularized skeletal muscle with minced muscle autografts improved recovery from volumetric muscle loss injury in a rat model. Tissue Eng Part A 22(19-20):1151-1163

Lepper C, Partridge TA, Fan CM (2011) An absolute requirement for Pax7-positive satellite cells in acute injury-induced skeletal muscle regeneration. Development 138(17):3639-3646

Li MT, Willett NJ, Uhrig BA, Guldberg RE, Warren GL (2014) Functional analysis of limb recovery following autograft treatment of volumetric muscle loss in the quadriceps femoris. J Biomech 47(9):2013-2021

Mase VJ Jr, Hsu JR, Wolf SE, Wenke JC, Baer DG, Owens J, Badylak SF, Walters TJ (2010) Clinical application of an acellular biologic scaffold for surgical repair of a large, traumatic quadriceps femoris muscle defect. Orthopedics 33(7):511

Podesser BK, Rinaldi M, Yona NA, Pulpon LA, Villemot JP, Haverich A, Duveau D, Brandrup-Wognsen G, Gronda E, Costard-Jackle A, Crespo-Leiro MG, Khazen CS, Vigano M, Segovia J, Mattei MF, Harringer W, Treilhaud M, Karason K, Mangiavacchi M, Laufer G (2005) Comparison of low and high initial tacrolimus dosing in primary heart transplant recipients: a prospective European multicenter study. Transplantation 79(1):65-71
Pollot BE, Corona BT (2016) Volumetric Muscle Loss. Methods Mol Biol 1460:19-31 Pollot BE, Goldman SM, Wenke JC, Corona BT (2016) Decellularized extracellular matrix repair of volumetric muscle loss injury impairs adjacent bone healing in a rat model of complex musculoskeletal trauma. J Trauma Acute Care Surg 81(5 Suppl 2 Proceedings of the 2015 Military Health System Research Symposium):S184-S190

Rivera JC, Corona BT (2016) Muscle-related disability following combat injury increases with time. US Army Med Dep J. Jan-Mar:30-34

Sadtler K, Estrellas K, Allen BW, Wolf MT, Fan H, Tam AJ, Patel CH, Luber BS, Wang H, Wagner KR, Powell JD, Housseau F, Pardoll DM, Elisseeff JH (2016) Developing a pro-regenerative biomaterial scaffold microenvironment requires T helper 2 cells. Science 352(6283):366-370

Sulaiman OA, Voda J, Gold BG, Gordon T (2002) FK506 increases peripheral nerve regeneration after chronic axotomy but not after chronic schwann cell denervation. Exp Neurol 175(1):127-137

Thomson AW, Bonham CA, Zeevi A (1995) Mode of action of tacrolimus (FK506): molecular and cellular mechanisms. Ther Drug Monit 17(6):584-591

Tidball JG (2005) Inflammatory processes in muscle injury and repair. Am J Physiol Regul Integr Comp Physiol 288(2):R345-R353

Ward CL, Ji L, Corona BT (2015) An Autologous muscle tissue expansion approach for the treatment of volumetric muscle loss. Biores Open Access 4(1):198-208

Ward CL, Pollot BE, Goldman SM, Greising SM, Wenke JC, Corona BT (2016) Autologous minced muscle grafts improve muscle strength in a porcine model of volumetric muscle loss injury. J Orthop Trauma 30(12):e396-e403

Willett NJ, Li MT, Uhrig BA, Boerckel JD, Huebsch N, Lundgren TL, Warren GL, Guldberg RE (2013) Attenuated human bone morphogenetic protein-2mediated bone regeneration in a rat model of composite bone and muscle injury. Tissue Eng Part C Methods 19(4):316-325

\section{Submit your manuscript to a SpringerOpen ${ }^{\circ}$ journal and benefit from:}

- Convenient online submission

- Rigorous peer review

- Open access: articles freely available online

- High visibility within the field

Retaining the copyright to your article

Submit your next manuscript at springeropen.com 